\title{
BMJ Open Challenges of diagnosis and management of giant cell arteritis in general practice: a multimethods study
}

\author{
Toby Helliwell, ${ }^{1}$ Sara Muller, ${ }^{1}$ Samantha L Hider, ${ }^{1,2}$ James A Prior, ${ }^{1}$ \\ Jane C Richardson, ${ }^{1}$ Christian D Mallen ${ }^{1}$
}

To cite: Helliwell T, Muller S, Hider SL, et al. Challenges of diagnosis and management of giant cell arteritis in general practice: a multimethods study. BMJ Open 2018;8:e019320. doi:10.1136/ bmjopen-2017-019320

- Prepublication history for this paper is available online. To view these files, please visit the journal online (http://dx.doi. org/10.1136/bmjopen-2017019320).

Presented as a poster at BSR Conference 2015.

Received 25 August 2017 Revised 2 November 2017 Accepted 4 December 2017

Check for updates

${ }^{1}$ Arthritis Research UK Primary Care Centre, Primary Care Sciences, Keele University, Keele, UK

${ }^{2}$ Department of Rheumatology, Haywood Rheumatology Centre, Keele, UK

Correspondence to

Dr Toby Helliwell;

t.helliwell@keele.ac.uk

\section{ABSTRACT}

Background In the UK, general practitioners (GPs) are usually the first medical contact for patients with suspected giant cell arteritis (GCA). While rare, it is critical not to miss, as delayed treatment can lead to significant complications including permanent visual loss. To date, little is known about the approach and challenges to diagnosis and management of GCA by GPs.

Objective To investigate the diagnosis and management of patients with suspected GCA in UK general practice.

Design and participants A multimethods approach was taken, comprising a postal survey of 5000 randomly selected UK GPS and semistructured telephone interviews of $24 \mathrm{GPs}$ from across the UK.

Setting UK general practice.

Results 1249 questionnaires were returned. 879 responders $(70 \%)$ indicated that they had diagnosed and managed a patient with GCA. A variety of clinical features were used to identify GCA. $21.9 \%$ suggested that they would exclude GCA as a diagnosis if headache was absent and around one-third do not routinely initiate glucocorticoid treatment prior to referral. Significant regional variations in referral pathways were reported. Thematic analysis of interview transcripts highlighted fears relating to a missed diagnosis of GCA and the non-specific nature of early GCA presentation. Accessing specialist care was highlighted as challenging by many GPs and that a national standard fast-track pathway is lacking to support this patient group. Additionally there were significant concerns regarding potential adverse effects relating to long-term treatment with glucocorticoids.

Conclusion GPs appear to over-rely on headache to identify GCA and marked geographical differences in management, with conflicting referral pathways and difficulties in accessing appropriate services exist in the UK. A national standard for fast-tracking patients with suspected GCA to relevant specialists would be beneficial to improve care and outcomes for patients with GCA.

\section{BACKGROUND}

Giant cell arteritis (GCA) is the most common large/medium vessel vasculitis. ${ }^{1}$ It is strongly associated with polymyalgia rheumatica (PMR) with an estimated incidence of 1.0 per 10000 patient-years. ${ }^{2}$ Barraclough et al estimated that a full-time general practitioner (GP) will see one new case of GCA every 1-2

\section{Strengths and limitations of this study}

- Multimethods approach, allowing the identification of significant challenges relating to giant cell arteritis (GCA) management in primary care and subsequent in-depth exploration of those issues.

- First large study to investigate diagnosis and management of GCA in general practice.

- Suboptimal response rate and therefore potential lack of generalisability of findings although responder demographics of the questionnaire study relating to age, gender and general practitioner (GP) role were comparable to national GP demographics.

- Telephone interviews often viewed as inferior to face-to-face interviews for qualitative studies.

- There is the potential for discrepancies between reported behaviour and actual behaviour, this is inherent in both survey and interview studies.

years, although this will greatly depend on practice population demographics. ${ }^{3}$

Classical presenting features of GCA include new-onset headache or head pain (which may be unilateral and often temporal), scalp tenderness, jaw and tongue claudication, constitutional and visual symptoms. ${ }^{1}$ Usually, there is a significant inflammatory response with raised inflammatory markers. However, it can present atypically which may lead to delays in diagnosis and potentially irreversible complications such as sight loss. ${ }^{4}$ Once GCA is suspected, treatment with high dose glucocorticoids (often prednisolone in the UK) should be initiated along with early specialist referral to confirm diagnosis and prevent potential disease complications. ${ }^{1}$

Patients with suspected GCA are usually identified clinically, followed by specialist referral for temporal artery biopsy (TAB) to confirm diagnosis. However, ultrasound scanning has been shown to be a useful and non-invasive tool to help diagnose $\mathrm{GCA}^{5}$ although typical ultrasound features of GCA may diminish after just a few days of glucocorticoid treatment, whereas histological features 
of GCA may still be evident on TAB several months after initiation of treatment. ${ }^{6}$ However, the sensitivity of TAB can vary with $13 \%-19 \%$ of patients with typical features of GCA having a negative TAB. ${ }^{7}$

In the UK, GPs are the first point of medical contact for most patients. The role of the GP involves maintaining a high index of suspicion for the disorder, to initiate early therapy and urgently refer to an appropriate specialist for diagnostic confirmation. ${ }^{1}$ Following diagnosis, GPs are often key in tapering glucocorticoid treatment as well as monitoring and management of glucocorticoid-related adverse effects and impact on comorbidity, for example, osteoporosis, cardiovascular disease, diabetes and development of serious infections. ${ }^{28}$

The aim of this study was to investigate the diagnostic challenges and initial and ongoing management of patients with GCA by GPs in the UK.

\section{MATERIALS AND METHODS}

Given the potential variation in management practices due to multiple influences, such as patient presentation, multimorbidity, availability of services and variations in practice and local policy, a multimethods approach combining two study methodologies was chosen to produce a more complete overall description of current GP diagnostic and management practices for GCA. ${ }^{9}$ First, a national cross-sectional postal survey of 5000 randomly selected UK GPs was undertaken to investigate PMR and its closely associated illness of GCA, followed by a semistructured telephone interview study with a purposive sample of survey responders to investigate in depth the challenges of diagnosis and management associated with GCA and PMR. The cross-sectional postal survey was undertaken first, with the findings used to help develop the topic guide for the interview study. This paper presents the combined findings from the two studies relating to GCA.

\section{PMR national cross-sectional postal questionnaire survey}

A cross-sectional survey was mailed to a random sample of 5000 GPs from across the UK identified from the Binley's database. The Binley's database contains the names and addresses of the majority of GPs working in the UK. It also contains other forms of information, including the type of practice, the practice population size, practitioner seniority and some of the clinical services provided (www.binleys.com). An online option for survey completion was also available through Survey Monkey (https:// www.surveymonkey.com). Non-responders were sent a reminder card after 2 weeks and a further survey pack after 4 weeks. The survey was closed 6 weeks after the second survey pack was sent.

No standard survey instrument exists for assessing diagnosis and management of GCA by GPs and so questions were specifically developed using current literature and guidelines for GCA. ${ }^{1}$ Questions related to how diagnosis was made (signs and symptoms) and how the GP managed patients with suspected GCA. A mixture of open and closed response questions were used. The questionnaire was piloted among GPs, rheumatologists and patients.

Descriptive statistics were generated (mean, SD and IQR) using the statistical analysis package SPSS V.22.0 for closed response questions(IBM). For open response questions, a thematic content analysis was used. ${ }^{10}$

\section{The interview study}

Participants in the interview study were purposively sampled from responders to the GP survey who had agreed to further contact. To reflect as broad a range of practitioner experience as possible, sampling was based on clinical experience, gender and clinical seniority. The qualitative interview study topic guide which was used as a guide for topics to discuss, was informed by findings from the cross-sectional survey and relevant GCA literature. The topic guide was reviewed and refined with feedback from GPs, rheumatologists and qualitative researchers. As transcripts were reviewed, the topic guide was modified to focus on themes identified from early interviews. The topic guide was piloted with two GPs and refined within the research team. These interviews were not included in the data analysis.

Interviews were audio recorded and transcribed verbatim using an approved transcription company. ${ }^{11}$ The resulting transcripts were screened to remove any identifying information. Thematic analysis, as described by Braun and Clarke, was used to analyse resulting transcript data. ${ }^{12}$ Analysis of the transcripts was managed using NVivo V.10. ${ }^{13}$ TH performed the analysis and an inter-rater exercise was undertaken in which three other researchers (SM, SLH, JCR) were asked to independently analyse and identify general themes relating to a randomly selected interview to compare with findings by TH. No changes resulted from this exercise.

\section{RESULTS}

One thousand two hundred and forty-nine (25\%) completed questionnaires were received and analysed. Eight hundred and seventy-nine (70\%) GPs had indicated that they had managed a patient with GCA. Responders to the survey had a mean age of 44 years (SD 9.25) and a mean of 13.5 years since qualifying as a GP. Fifty-two per cent were female and the majority were partners (74\%), with salaried (21\%) and locum GPs (3\%) comprising the remainder. For the qualitative study, 24 GP participants were telephone interviewed from various regions across the UK. Sixteen participants were female and 15 participants were GP partners.

\section{Questionnaire survey: initial diagnosis and management}

Free text open response questions in the questionnaire were used to ask all participants to describe how they made a diagnosis of GCA. The results are summarised in table 1 . 
Table 1 The features used by responders to identify GCA

\begin{tabular}{lc} 
GCA feature & Theme frequency \\
\hline Headache/head symptoms & 1071 \\
Visual disturbances & 671 \\
$\begin{array}{l}\text { Scalp (including temporal artery } \\
\text { tenderness) }\end{array}$ & 468 \\
Jaw symptoms & 420 \\
PMR symptoms & 69 \\
Systemic symptoms & 65 \\
Fatigue & 29 \\
Joint/muscle symptoms & 20 \\
Tongue symptoms & 12 \\
\hline
\end{tabular}

GCA, giant cell arteritis; PMR, polymyalgia rheumatica.

The predominant reported clinical feature used to diagnose GCA was headache, along with visual disturbance and scalp tenderness. Survey responders indicated that they often used a combination of features when making a new diagnosis. Of particular note, however, was that $21.9 \%$ of responders indicated that they only use headache to identify GCA.

Management of GCA can be divided into (1) initial treatment and referral and (2) long-term glucocorticoid reduction and monitoring. For GPs, initial management is intimately associated with diagnosis as patients with suspected GCA require urgent specialist referral for definitive diagnosis and treatment. Table 2 summarises the immediate subsequent actions of GPs who have identified patients with suspected GCA.

Guidance advises that treatment should not be delayed and that appropriate urgent referral for specialist diagnostic confirmation should be made. ${ }^{1}$ Four hundred and forty-five responders to the survey $(35.6 \%)$ indicated that they would not routinely initiate glucocorticoid treatment prior to referral. However, $78.7 \% \quad(\mathrm{n}=983)$ reported that if they were to initiate treatment, appropriate doses of between 40 and $60 \mathrm{mg}$ of prednisolone would be prescribed. GP responders indicated that they were referring patients with suspected GCA to a variety of

Table 2 Actions undertaken by GPs with patients with suspected GCA

\begin{tabular}{ll} 
Action & $\mathbf{n ~ ( \% )}$ \\
$\begin{array}{l}\text { Urgent blood tests, initiate treatment and refer } \\
\text { for outpatient review urgently, if blood tests }\end{array}$ & $554(44.4)$ \\
positive & \\
$\begin{array}{l}\text { Refer to hospital immediately without } \\
\text { investigation }\end{array}$ & $244(19.5)$ \\
$\begin{array}{l}\text { Urgent blood tests and refer to hospital } \\
\text { immediately if positive }\end{array}$ & $201(16.1)$ \\
$\begin{array}{l}\text { Urgent blood tests, initiate treatment and refer } \\
\text { for outpatients review routinely }\end{array}$ & $66(5.3)$ \\
\hline
\end{tabular}

GCA, giant cell arteritis; GP, general practitioner.
Table 3 Specialties to which patients with GCA are referred

\begin{tabular}{lc}
\hline Specialty & Frequency (\%) \\
\hline Rheumatology & $478(38.3)$ \\
Ophthalmology & $366(29.3)$ \\
General medicine & $144(11.5)$ \\
Accident and emergency & $35(2.8)$ \\
Neurology & $12(1.0)$ \\
Elderly care & $9(0.7)$ \\
Other & $41(3.3)$ \\
Missing & $164(13.1)$ \\
\hline
\end{tabular}

GCA, giant cell arteritis.

different specialities using an assortment of referral pathways, depending on the geographical location in the UK. Table 3 summarises to which specialty survey responders refer patients with suspected GCA.

\section{Themes identified from the qualitative study Diagnosis}

The two main themes identified from the interview study related first to the presenting features of GCA and second to fears of missing a diagnosis of GCA.

\section{Presenting features of GCA}

When asked about GCA symptoms in the interviews, participants often gave textbook descriptions of classical features of GCA.

Headache in someone over 55 you think giant cell arteritis really, that's my mantra, new different headache, classically unilateral but not always, focused around the temple, potentially some tenderness there, possibly protruding temporal artery, classically tender when they're combing their hair, but also looking for things like jaw claudication or tongue symptoms, $[\ldots]$ and obviously the dread of visual disturbance as well really which can be anything really. GP6 $(20, F, P)^{\mathrm{i}}$

While textbook descriptions of classical GCA were given, there was recognition that some of these features may be difficult to recognise or link to GCA.

jaw claudication is interesting, because I know at the time, my colleague and myself, kind of, looked a bit more up about GCA and he said, 'I've never heard of jaw claudication. GP15 (25, F, P)

\section{Fear of missing case of GCA}

GPs expressed considerable fear about missing a diagnosis of GCA given the potential for irreversible visual loss.

${ }^{\mathrm{i}}$ Key: GP identifier (time qualified as a GP (years), gender (male/ female), seniority/role (S, salaried; P, partner;)). 
I find it, sort of, trickier, I think, to diagnose. I worry about it more. I worry about missing it. And I feel far less confident about treating it. I think when I was first qualified as a GP I think I thought somebody had got it every week. Anybody who'd got a headache, you know. GP24 (12, F, S)

Fears surrounding missing a diagnosis of GCA also related to the potential for a wide range of symptoms and atypical presentations.

Just with a vague headache, and hadn't had any visual disturbance at that point in time. And he didn't really have a lot of temporal artery tenderness. [...] We did some bloods at that point, and the ESR and CRP were normal $[\ldots] \ldots$ I wrote in the notes at the time, 'Excludes GCA'... which, having read a bit more about it since, after this happened, doesn't totally exclude it. GP15 (25 , F, P)

\section{Management}

Two main themes were identified from the interview transcripts. First, initial and ongoing treatment and monitoring and second, referral for definitive diagnostic confirmation by a specialist.

\section{Initial and ongoing treatment}

Most participants indicated that they would initiate patients with suspected GCA on appropriate doses of prednisolone.

I think the rheumatologists would say start the $60[\mathrm{mg}]$ and I will see them in clinic. GP4 (6, M, P)

However, there was recognition that treatment could impact on the sensitivity of the $\mathrm{TAB}$, especially if it was going to be delayed.

what then happens in secondary care, it's less than ideal, they seem to rotate who might do a temporal artery biopsy between vascular, ophthalmology and general surgery $[\ldots]$ but the patient generally is having that temporal biopsy before ever seeing a rheumatologist and the timeliness of that temporal artery biopsy is not ideal. GP6 (20, F, P)

Local policy also had a significant impact on how patients with suspected GCA were initially treated. This, as well as concerns surrounding the impact that treatment could have on biopsy effectiveness, may account for some of the significant number of participants who indicated that they would not initiate treatment prior to referral.

Locally this gets referred to ophthalmology [...] and our practice is actually within the grounds of the hospital so we've got no issues really in terms of administering steroids you know before they were seen, they would be seen within an hour by an ophthalmologist. GP7 (10, M, P)
The principal and overarching concern relating to long-term management was the potential adverse effects of glucocorticoid treatment.

Well, it's a good two years of treatment with steroids and all the complications and side effects that they carry with them. So, yes, and high doses of it, which have been poorly tolerated with the patients. [...] One patient, she had diabetes, and she was started on the steroids, and she was struggling with awful side effects from the steroids. She developed, well, lots of depressive symptoms. Her blood sugars went all over the place. She got a lot of pitting oedema of the legs, which was hampering her mobility. She got unsteadiness due to the steroids. GP21 (7, F, S)

\section{Referral for definitive diagnostic confirmation by a specialist}

Specialist referral for definitive diagnostic confirmation was a significant issue for GPs, with referral pathways being highly variable across the UK. The specialty to which patients with suspected GCA were referred can depend on presenting clinical features; however, some of this variation reflects local policy and also the regional availability of services and specialities.

If their history was suspicious and their inflammatory markers were raised, I would then contact...well we've had this issue between rheumatology and ophthalmology and who to contact, and the line seems to be that if they've got any visual symptoms then they go to ophthalmology and if they haven't then they go to rheumatology. GP13 (5, F, S)

However, in some regions of the UK GPs reported that referral pathways were not clear and that specialist referral can be challenging.

But, generally, you speak to the on-call medical team, and they will advise me to speak to someone else. And then they advise me to speak to someone else. So you end up making loads of phone calls to try and find out which route you go in. GP21 (7, F, S)

Some participants reported that their local policy involved the GP requesting the TAB prior to review by a specialist. This often created challenges in itself.

we would try and get a temporal artery biopsy fairly promptly. It has been a bit difficult in the past, and you know, you're supposed to get it done within a day or two. We traipse round the ophthalmologists, who say, 'No, speak to the vascular people.' Who say, 'No, speak to the general surgeons.' Well, we tried, initially, referring to the ophthalmologist, and they just aren't keen at all [...] at the moment we've had, a general surgery team who have done a temporal artery biopsy for us, and the vascular surgeons have. GP15 (25, F, P)

Further quotations illustrating the two main themes can be seen in table 4 . 
Table 4 Verbatim quotations from the qualitative study and open responses from the cross-sectional survey

\begin{tabular}{|c|c|c|}
\hline Theme & Subtheme & Verbatim quotation \\
\hline Challenges of diagnosis & $\begin{array}{l}\text { Fear of missing GCA } \\
\text { and non-specific } \\
\text { presentation }\end{array}$ & $\begin{array}{l}\text { "an elderly lady who was having headaches and kind of pain around } \\
\text { her eyes and l'm trying to think what other symptoms she had, general } \\
\text { misery really. And it sort of came and went and came and went and } \\
\text { she didn't really have any visual problems which is good and when you } \\
\text { said to her, 'Does it hurt to chew?' she'd say, 'Oh yes I think it does'. } \\
\text { And so yes all of that so in the end I started, I did discuss it with our } \\
\text { local physicians because just in that situation where you don't want to } \\
\text { miss it but on the other hand it doesn't seem like it's probably the most } \\
\text { likely diagnosis. And we got as far as them saying, 'Well if it's maybe } \\
\text { a possibility then go ahead and treat with steroids', at which point she } \\
\text { said, 'No I'm feeling much better thank you'. And that was that until she } \\
\text { started complaining about it again another few months later." GP17 (11, } \\
\text { F, P) }\end{array}$ \\
\hline
\end{tabular}

Initial and ongoing Starting treatment

treatment and monitoring

"And, certainly, in the past couple of years, we've started them on steroids first, because, kind of, getting anybody to see them quickly, you know, within a day or two, not been possible, which doesn't seem very ideal to me. And we've taken the view if it turns out to be wrong, we can stop it, but if we don't start it, there might be a problem before they get the biopsy. So that's, kind of, what we've done here." GP15 $(25, \mathrm{~F}, \mathrm{P})$

"Yes again just I think in terms of the ongoing management really because my experience with another patient, the one that ended up with visual disturbance, she sort of then fell between ophthalmology and rheumatology without either necessarily taking full responsibility for her and actually she was a patient of a partner of mine so he was kind of following her up but his experience was that he was piggy in the middle really." GP 6 (20, F, P)

Expediency of review "you refer them under a two week wait, and it's not that much of an emergency, whereas we all thought you referred them acutely, because it was that much of an emergency. So there was a big discrepancy of views between what we felt we'd been taught about it, and what other people were now doing." GP23 $(12, \mathrm{~F}, \mathrm{~S})$

"I know we, kind of, all get it drummed into us, you know, we should all get these things sent in on the day. But I think, well, one of them was hanging round for a year, and he didn't really come to any harm, except undue pain and distress that he had. And the other one was hanging round for a couple of months, you know. And they were both provedas I say, I'm turning the clock back 15 years - but I think they were both proven to be temporal arteritis. It maybe isn't that, kind of, you know, you must get them in on the day, as I thought as a medical student, you know." GP22 (15, M, P)

Challenges with referral Delays in temporal for definitive diagnostic artery biopsy confirmation by specialist
"The patient that I referred on the NHS, she ended up having a biopsy before she saw a consultant rheumatologist. So, yes, it was done that way round. The biopsy, of course, came back negative because the two week delay before getting it done meant the steroids had treated it." GP21 $(7, \mathrm{~F}, \mathrm{~S})$

"Local issue regarding whether ophthalmology or vascular surgery will perform temporal artery biopsy, reliability of this procedure and steroid response whilst waiting for the biopsy." Participant 2506 (4, 2, P)

Key to participant demographic: GP (n) (qualitative study identifier), Participant (n) (survey identifier) (time qualified as a GP (years), gender (Male/Female), seniority/role (S, salaried; P, partner)).

GCA, giant cell arteritis; GP, general practitioner.

\section{DISCUSSION}

This is the first study of its kind to explore diagnosis and a critical aspect of the care pathway for patients with suspected GCA. GCA remains a diagnosis that GPs worry about missing. While GPs were comfortable with classical presentation patterns, an over-reliance on headache to trigger consideration of a diagnosis of GCA was evident, with some GPs having limited awareness of the full range of symptoms associated with GCA. 
The predominant findings from the cross-sectional study, suggest initiation of treatment for patients with suspected GCA is not always routine. However, a significant finding from both the cross-sectional survey and qualitative interview study demonstrated that referral pathways across the UK vary greatly. Additionally, GPs have significant concerns relating to treatment with longterm glucocorticoids in this patient group, especially in conjunction with coexisting multimorbidity.

\section{Recommendations}

Early identification, referral and initiation of appropriate treatment for patients with suspected GCA in general practice is critical to prevent complications such as irreversible visual loss. ${ }^{1}$

GPs responding to the survey seem to be over-relying on headache when diagnosing GCA. Given that almost half of patients do not present with a classical temporal headache and that $24 \%$ of patients with proven GCA have no headache symptoms at all, ${ }^{14}$ excluding GCA on the basis of no headache has the potential to miss a significant proportion of patients with GCA. However, symptoms like headache are common ${ }^{15}$ with over half of over 65 year olds having had a headache in the previous 12 months, ${ }^{16}$ yet serious pathology is rare in general practice. Therefore the collective clinical picture needs to be considered and has to include the full range of features of GCA.

The group of patients with no headache are recognised to be at higher risk of permanent visual loss as a result of delayed diagnosis. ${ }^{47}$ Therefore, if alternative presentations are not recognised by GPs, they will continue to remain a high-risk group. Educating clinicians about other presenting symptoms and atypical presentations is essential to optimise diagnosis and reduce delays in instigating appropriate treatment and referral, which could reduce the potential for visual loss and serious long-term complications for this patient group.

A considerable proportion of GPs indicated that they would not initiate treatment prior to referral for specialist review. From the questionnaire responses, current primary care practice would seem to be in line with UK recommendations, ${ }^{1}$ indicating that appropriate doses of glucocorticoids, when given, are being prescribed at initiation. Additionally, there seems to be wide variation in practice across the UK relating to routes of referral and who arranges and performs TAB. Rheumatology remains the predominant specialty to whom GPs refer patients with suspected GCA, but a range of different specialities were identified by participants. These findings may in part reflect variations in local policy and the availability of specialities regionally. However, it may also identify a lack of GP awareness of current national GCA guidelines.

Research into conditions such as rheumatoid arthritis highlights that delays in diagnosis can occur at several points in the patient journey. These include the patient recognising that there is a problem requiring consultation (patient level), the patient then getting an appointment with the GP, the GP recognising that referral is needed and making the referral (GP delay) and the patient getting an appointment with the specialist (specialist delay). ${ }^{18}$ These points of delay are also likely to be relevant to patients with GCA. Health promotion could be used to improve patients' awareness of GCA, but may be of limited value given the rarity of the condition and the wide and non-specific symptoms that patients experience early in the disease course. However, educational strategies for front line clinicians in the early recognition and management of GCA is critical; although some responders indicated that there do not appear to be robust fast-track clinical pathways in their local region for patients with suspected GCA. Given the relative rarity of GCA and variation in its early presentation, ${ }^{14}$ the potential for it not being recognised or for initial misdiagnosis is high. In regions where TAB is arranged by the GP or undertaken before seeing the relevant specialist, there is the possibility that a significant amount of unnecessary biopsies are being performed especially given that there is a great burden of temporal artery biopsies on surgical departments with sometimes low yield rates of positive biopsy. ${ }^{19}$ This potentially could be avoided if patients with suspected GCA were carefully selected by a clinician with significant experience in diagnosing and identifying suspected GCA. No participants discussed temporal artery ultrasound which can be used to help identify patients with $\mathrm{GCA}^{5}$ and this may be because this imaging modality where available, is requested by the treating specialist and not the GP. Ultrasound techniques may be a preferred option for those with significant comorbidities or too frail to undergo biopsy but will have to be rapidly available to clinicians given the importance of starting glucocorticoid treatment in GCA and the rapid effects treatment has on typical ultrasound features. ${ }^{6}$ It is, however, less invasive and could be appropriate to being embedded in a care pathway for the rapid assessment of GCA in primary care to streamline patient pathways to help reduce diagnostic confusion, or to better identify patients for $\mathrm{TAB}^{20}$ thereby improving outcomes for patients with GCA. ${ }^{21}{ }^{22}$ Further, studies are being conducted to determine whether availability and accuracy of temporal artery ultrasound will alter requirements for biopsy. ${ }^{23}$

Delays in assessment for definitive diagnosis creates several conundrums surrounding initial treatment. Current guidance is clear that treatment should not be delayed and should be initiated in patients with suspected GCA, although the sensitivity of TAB declines the longer treatment has been given before biopsy. ${ }^{24}$ High dose glucocorticoid treatment may have a significant impact on symptoms by the time they present to the reviewing specialist and therefore definitive diagnosis for patients who have had a negative biopsy can become extremely challenging. However, an accurate diagnosis is critical and a decision to delay treatment in patients with true GCA could result in visual loss. Equally, a decision to continue treatment in someone who does not truly have GCA will expose that patient to an inappropriate treatment course 
(and therefore associated potential adverse effects) of glucocorticoids, as treatment often continues for many patients, despite a negative TAB. ${ }^{24}$

Our quantitative data suggested that over one-third of participants would not initiate treatment prior to referral despite UK national guidance which advises the immediate initiation of high dose glucocorticoids. ${ }^{1}$ This represents an area where further education to encourage the immediate initiation of treatment could improve outcomes for patients with GCA. However, not initiating treatment may be in part due to variations in local or practice policy.

\section{Strengths and limitations of this study}

The predominant strength of this study is the use of a multimethods approach, which has allowed the identification of significant challenges relating to GCA management in primary care and subsequent in-depth exploration of those issues.

The main weakness in this study was the suboptimal response rate and therefore the potential lack of generalisability of our findings. However, a response rate of $25 \%$ is comparable to similar musculoskeletal GP surveys conducted in the same setting. ${ }^{25}$ Additionally, while low response rates may increase the possibility of bias, responder demographics of the questionnaire study relating to age, gender and GP role were comparable to national GP demographics. ${ }^{26}$ The standard limitations of telephone interviews also apply in this study. While such interviews enabled participants to be interviewed from a wide geographical area and therefore generate rich data on differing local management policies, they may reduce rapport and non-verbal communication. However, the questions used in the topic guide were highly clinical and therefore the lack of rapport building or visual cues is unlikely to have significantly impacted on data quality, as participants were not revealing personal details. TH undertook all of the qualitative data analysis which potentially could impact on theme development due to personal preconceptions and misinterpretations. However, an inter-rater analysis was undertaken to ensure concordance of themes identified. This did not show any difference between raters. Finally, there is the potential for discrepancies between reported behaviour and actual behaviour, this is inherent in both survey and interview studies.

\section{CONCLUSION}

An increased focus on education and awareness of GCA (given its rarity and the range of presenting features including more subtle features such as limb claudication, constitutional symptoms, vascular bruits, asymmetry of pulses and or blood pressure, anaemia ${ }^{1}$ ) may aid better identification of patients with potential GCA. However, significant challenges around GCA remain in primary care, some of which need to be addressed in conjunction with specialist settings. National guidelines suggest that
GCA is a medical emergency and so treatment should not be delayed. However, as yet there are no UK national standardised fast-track referral/care pathways enabling rapid referral of patients suspected of having GCA yet fast-track pathways have been shown to potentially reduce the complication of sight loss in GCA. ${ }^{21}$ This study identifies wide variations in the way that patients are initially managed across the UK and therefore adopting standardised fast-track services for patients with suspected GCA could enable effective and accurate diagnosis and management and therefore improve outcomes for patients with GCA.

Acknowledgements We are grateful for the participation of all of the general practitioners who responded to the questionnaire and volunteered their precious time to participate in the telephone interviews. Special thanks go to Dr Antonia Middleditch and Dr Edward Jutsum for their help in interview and topic guide development. Acknowledgements are also given to the Research Institute for Primary Care and Health Sciences, Keele University and the professional services staff who supported the study.

Contributors Authors had access to all the study data, take responsibility for the accuracy of the analysis, and had authority over the manuscript and the decision to submit for publication. Guarantor of overall study integrity: TH and CDM. Study concept and design: TH, SM, SLH, JCR and CDM. Data collection and interpretation: TH, SM, SLH, JCR and CDM. Analyses: TH, SM, SLH, JCR and CDM. Manuscript preparation: TH, SM, SLH, JAP, JCR and CDM. Final approval of manuscript: TH, SM, SLH, JAP, JCR and CDM.

Funding This work was funded by an Arthritis Research UK Clinician Scientist Award awarded to CDM (19634). CDM is funded by the National Institute for Health Research (NIHR) Collaborations for Leadership in Applied Health Research and Care West Midlands, the NIHR School for Primary Care Research (SPCR) and an NIHR Research Professorship in General Practice (NIHR-RP-2014-04-026). TH is currently funded by an NIHR Clinical Lectureship in General Practice and was funded by an NIHR in practice fellowship and an NIHR School for Primary Care Research GP career progression award during the time that this research was undertaken. JAP is funded by a Launching Fellowship from the NIHR School for Primary Care Research.

Disclaimer The views expressed are those of the author(s) and not necessarily those of the NHS, the NIHR or the Department of Health.

Competing interests None declared.

Patient consent Obtained.

Ethics approval Ethical approval for both studies was granted by the Keele University Ethics Review Panel (qualitative study ERP178, survey ERP2206).

Provenance and peer review Not commissioned; externally peer reviewed.

Data sharing statement The datasets analysed during the current study may be available from the corresponding author on reasonable request.

Open Access This is an Open Access article distributed in accordance with the Creative Commons Attribution Non Commercial (CC BY-NC 4.0) license, which permits others to distribute, remix, adapt, build upon this work non-commercially, and license their derivative works on different terms, provided the original work is properly cited and the use is non-commercial. See: http://creativecommons.org/ licenses/by-nc/4.0/

(c) Article author(s) (or their employer(s) unless otherwise stated in the text of the article) 2018. All rights reserved. No commercial use is permitted unless otherwise expressly granted.

\section{REFERENCES}

1. Dasgupta B, Borg FA, Hassan N, et al. BSR and BHPR guidelines for the management of giant cell arteritis. Rheumatology 2010;49:1594-7.

2. Petri $H$, Nevitt $A$, Sarsour $K$, et al. Incidence of giant cell arteritis and characteristics of patients: data-driven analysis of comorbidities. Arthritis Care Res 2015;67:390-5.

3. Barraclough K, Mallen CD, Helliwell T, et al. Diagnosis and management of giant cell arteritis. Br J Gen Pract 2012;62:329-30. 
4. Ezeonyeji AN, Borg FA, Dasgupta B. Delays in recognition and management of giant cell arteritis: results from a retrospective audit. Clin Rheumatol 2011;30:259-62.

5. Niederkohr RD, Levin LA. A Bayesian analysis of the true sensitivity of a temporal artery biopsy. Invest Ophthalmol Vis Sci 2007;48:675-80.

6. Schmidt WA. Role of ultrasound in the understanding and management of vasculitis. Ther Adv Musculoskelet Dis 2014;6:39-47.

7. Breuer GS, Nesher R, Nesher G. Negative temporal artery biopsies: eventual diagnoses and features of patients with biopsy-negative giant cell arteritis compared to patients without arteritis. Clin Exp Rheumatol 2008;26:1103-6.

8. Mohammad AJ, Englund M, Turesson C, et al. Rate of Comorbidities in Giant Cell Arteritis: A Population-based Study. J Rheumatol 2017;44:84-90.

9. Creswell JW, Fetters MD, Ivankova NV. Designing a mixed methods study in primary care. Ann Fam Med 2004;2:7-12.

10. Krippendorf K. Content analysis. Barbnouw E, International encyclopaedia of communication. New York: Oxford University Press, 1989:403-7.

11. The Transcription Company. http://thetranscription.co.uk/

12. Braun V, Clarke V. Using thematic analysis in psychology. Qual Res Psychol 2006;3:77-101.

13. NVivo qualitative data analysis Software;QSR International Pty Ltd Version 10, 2012

14. Smetana GW, Shmerling RH. Does this patient have temporal arteritis? JAMA 2002;287:92-101.

16. Prencipe M, Casini AR, Ferretti C, et al. Prevalence of headache in an elderly population: attack frequency, disability, and use of medication. Journal of neurology. Neurosurgery2001;70:377-81.
15. Boardman HF, Thomas E, Millson DS, et al. One-year follow-up of headache in an adult general population. Headache 2005;45:337-45.

17. Prior JA, Ranjbar H, Belcher J, et al. Diagnostic delay for giant cell arteritis - a systematic review and meta-analysis. BMC Med 2017;15:120.

18. Raza K, Stack R, Kumar K, et al. Delays in assessment of patients with rheumatoid arthritis: variations across Europe. Ann Rheum Dis 2011;70:1822-5.

20. Ball EL, Walsh SR, Tang TY, et al. Role of ultrasonography in the diagnosis of temporal arteritis. Br J Surg 2010;97:1765-71.

19. Cristaudo AT, Mizumoto R, Hendahewa R. The impact of temporal artery biopsy on surgical practice. Ann Med Surg 2016;11:47-51.

21. Patil P, Williams M, Maw WW, et al. Fast track pathway reduces sight loss in giant cell arteritis: results of a longitudinal observational cohort study. Clin Exp Rheumatol 2015;33.

22. Diamantopoulos AP, Haugeberg G, Lindland A, et al. The fasttrack ultrasound clinic for early diagnosis of giant cell arteritis significantly reduces permanent visual impairment: towards a more effective strategy to improve clinical outcome in giant cell arteritis? Rheumatology 2016;55:66-70.

23. Luqmani R, Lee E, Singh S, et al. The Role of Ultrasound Compared to Biopsy of Temporal Arteries in the Diagnosis and Treatment of Giant Cell Arteritis (TABUL): a diagnostic accuracy and costeffectiveness study. Health Technol Assess 2016;20:1-238.

24. Pieri A, Milligan R, Hegde V, et al. Temporal artery biopsy: are we doing it right? Int J Health Care Qual Assur 2013;26:559-63.

25. Clarson LE, Nicholl BI, Bishop A, et al. Monitoring Osteoarthritis: A Cross-sectional Survey in General Practice. Clin Med Insights Arthritis Musculoskelet Disord 2013;6:CMAMD.S12606-91.

26. NHS Digital Internet. UK national information, data and IT systems for health and care services. (cited 25 Aug 2017). 